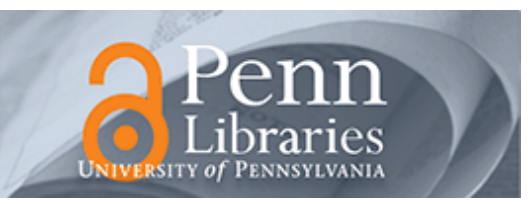

University of Pennsylvania ScholarlyCommons

8-16-2011

\title{
Effect of Electric and Stress Field on Structures and Quantum Conduction of $\mathrm{Cu}$ Nanowires
}

C. $\mathrm{He}$

Xi'an Jiaotong University

Lin Qi

University of Pennsylvania

W. X. Zhang

Chang'an University

H. Pan

Xi'an Jiaotong University

Follow this and additional works at: https://repository.upenn.edu/mse_papers

Part of the Materials Science and Engineering Commons

\section{Recommended Citation}

He, C., Qi, L., Zhang, W. X., \& Pan, H. (2011). Effect of Electric and Stress Field on Structures and Quantum Conduction of Cu Nanowires. Retrieved from https://repository.upenn.edu/mse_papers/213

He, C., Qi, L., Zhang, W.X., Pan, H. (2011). Effect of electric and stress field on structures and quantum conduction of Cu nanowires. Applied Physics Letters. 99, 073105.

(C) 2011 American Institute of Physics. This article may be downloaded for personal use only. Any other use requires prior permission of the author and the American Institute of Physics. The following article appeared in Applied Physics Letters and may be found at http://dx.doi.org/10.10631/1.3626841.

This paper is posted at ScholarlyCommons. https://repository.upenn.edu/mse_papers/213

For more information, please contact repository@pobox.upenn.edu. 


\title{
Effect of Electric and Stress Field on Structures and Quantum Conduction of $\mathrm{Cu}$ Nanowires
}

\author{
Abstract \\ The ballistic transport properties of Cu nanowires under different electric and stress fields are \\ investigated for future application in microelectronics using first-principles density-function theory. \\ Relative to the case with the electric field only, the stability and quantum conduction of both nonhelical \\ and helical atomic strands are enhanced by applying a stress field $\mathrm{F}$. Under $V=1 \mathrm{~V} / \AA$, the most excellent \\ quantum conductivity is exhibited at $F=1.5 \mathrm{nN}$ for the nonhelical atomic strands while at $F=2 \mathrm{nN}$ for the \\ helical ones, and the latter is more stable with collapse-resistant $F$ high as $3 \mathrm{nN}$ compared to the former \\ as $2 \mathrm{nN}$.

\section{Disciplines} \\ Engineering | Materials Science and Engineering

\section{Comments} \\ He, C., Qi, L., Zhang, W.X., Pan, H. (2011). Effect of electric and stress field on structures and quantum \\ conduction of Cu nanowires. Applied Physics Letters. 99, 073105. \\ (C) 2011 American Institute of Physics. This article may be downloaded for personal use only. Any other \\ use requires prior permission of the author and the American Institute of Physics. The following article \\ appeared in Applied Physics Letters and may be found at http://dx.doi.org/10.10631/1.3626841.
}




\title{
Effect of electric and stress field on structures and quantum conduction of Cu nanowires
}

\author{
C. He, ${ }^{1}$ L. Qi, ${ }^{2}$ W. X. Zhang, ${ }^{3}$ and H. Pan ${ }^{4, a)}$ \\ ${ }^{1}$ State Key Laboratory for Mechanical Behavior of Materials, School of Materials Science and Engineering, \\ Xi' an Jiaotong University, Xi' an 710049, China \\ ${ }^{2}$ Department of Materials Science and Engineering, University of Pennsylvania, Philadelphia, Philadelphia, \\ Pennsylvania 19104, USA \\ ${ }^{3}$ School of Materials Science and Engineering, Chang' an University, Xi' an 710064, China \\ ${ }^{4}$ Department of Environmental Science and Engineering, Xi' an Jiaotong University, Xi' an 710049, People's \\ Republic of China
}

(Received 31 May 2011; accepted 27 July 2011; published online 16 August 2011)

\begin{abstract}
The ballistic transport properties of $\mathrm{Cu}$ nanowires under different electric and stress fields are investigated for future application in microelectronics using first-principles density-function theory. Relative to the case with the electric field only, the stability and quantum conduction of both nonhelical and helical atomic strands are enhanced by applying a stress field F. Under $V=1 \mathrm{~V} / \AA$, the most excellent quantum conductivity is exhibited at $F=1.5 \mathrm{nN}$ for the nonhelical atomic strands while at $F=2 \mathrm{nN}$ for the helical ones, and the latter is more stable with collapse-resistant $F$ high as 3 $\mathrm{nN}$ compared to the former as $2 \mathrm{nN}$. (C) 2011 American Institute of Physics. [doi:10.1063/1.3626284]
\end{abstract}

There is an increasing interest in developing $\mathrm{Cu}$ nanowires (NWs) for possible applications in flexible displays and molecular electronic device. ${ }^{1-3}$ As the length and width scales of NWs are reduced to the mean free path of electrons, the electron transport mechanism changes from diffusive to ballistic. It is now known that the electric conductance is independent on the length of the NWs and the quantum conduction $G$ has been observed ${ }^{4}$ as predicted by the Landauer formula, ${ }^{5}$ where $G$ is quantized in units of $G_{0}=2 e^{2} / h$ where $e$ denotes the electronic amount and $h$ the Planck constant. Both atomic structures and size of NWs affect the transport properties. ${ }^{5-8}$ When NW is sufficient thin, beside the conventional structure, ${ }^{9}$ it can turn exotic and these structures should in fact be rather reading realized in nature. ${ }^{10}$ However, the effect of electric and stress field coupling on $G$, which is the precondition of the electronic transport, has not been considered systemically up to now.

In this letter, density functional theory (DFT) calculations are performed to determine the changes of atomic and electronic structures of $\mathrm{Cu}$ NWs in light of density of states (DOS) under stress and electric fields. In the calculations, two optimized atomic structures of $\mathrm{Cu}$ NWs are used as the starting points. It is found that atomic structures and related structure changes decide the size of $\mathrm{G}(V)$ function of $\mathrm{Cu}$ NWs, and the electronic distribution within NWs also reveals the magnitude of $\mathrm{G}$.

The DFT calculation is provided by $\mathrm{DMOL}^{3}$ code. ${ }^{11,12}$ The generalized gradient approximation is employed to optimize geometrical structures and calculate properties of $\mathrm{Cu}$ NWs with the Perdew-Burke-Ernzerhof correlation gradient correction. ${ }^{11,12}$ The all-electron relativistic Kohn-Sham wave functions are expanded in the local atomic orbital basis set. The $\mathrm{Cu}$ NWs are modeled in a tetragonal supercell with onedimensional periodical boundary conditions along the NWs.

\footnotetext{
a) Author to whom correspondence should be addressed. Electronic mail: panhua@xjtu.edu.cn.
}

Our outlines of the used structures are directly referred to the results of Wang et al. ${ }^{13}$ The length of $\mathrm{Cu}$ NWs $(L)$, which is determined by the distance of the projection of mean locations of atom centers in the 1st and 10th layers on the axis. The $k$-point is set to $5 \times 5 \times 1$ for all slabs, which brings out the convergence tolerance of energy of $2.0 \times 10^{-5} \mathrm{Ha}$ $(1 \mathrm{Ha}=27.2114 \mathrm{eV})$, maximum force of $0.004 \mathrm{Ha} / \AA$, and maximum displacement of $0.005 \AA$. $V$ is directly applied along the axis of $\mathrm{Cu} \mathrm{NW}$ with values of 0 and $1 \mathrm{eV} / \AA . G(V)$ values are determined by the Landauer formula. ${ }^{14}$ The layer

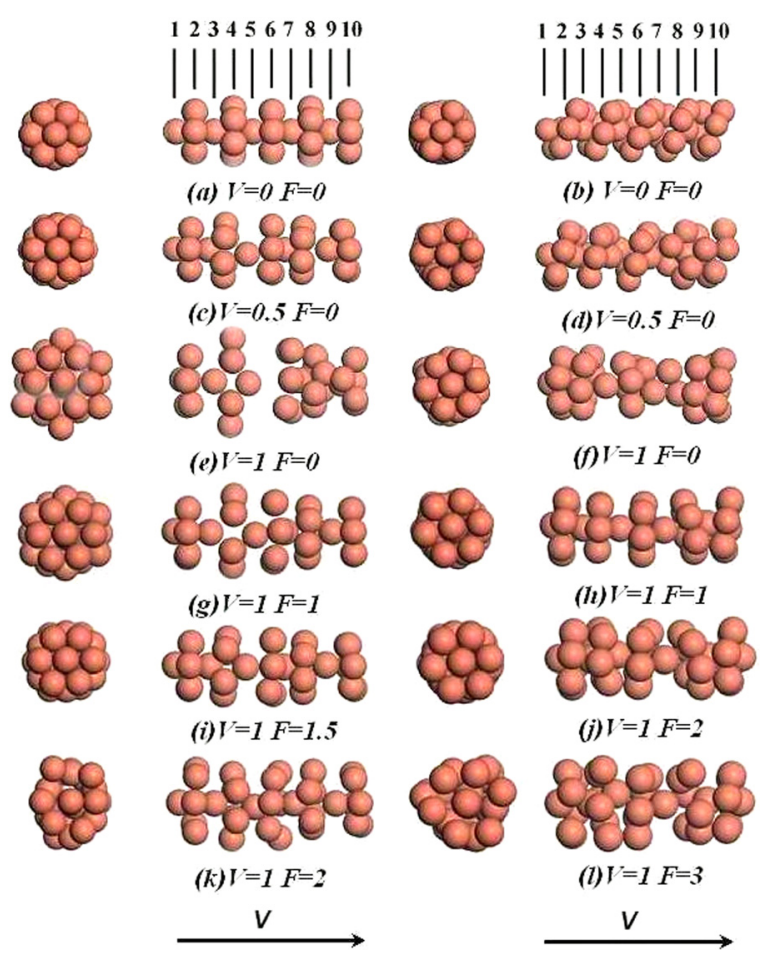

FIG. 1. (Color online) Morphologies of Cu NWs as a function of $V$ and $F . V$ in $\mathrm{VA}^{-1} . F$ in nN. The arrow shows the direction of $V$. 


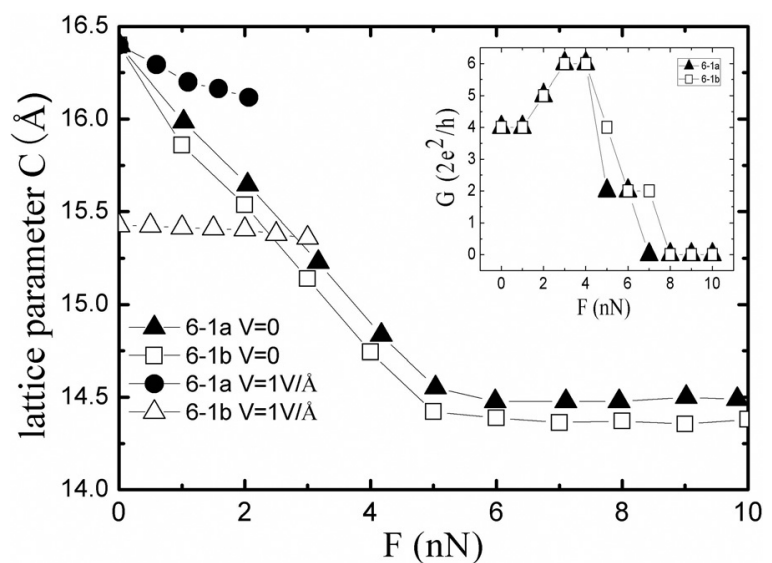

FIG. 2. The lattice parameter C (length) of Cu NWs $L(V=0, F)$ functions of 6-1a and 6-1b. The inset picture is $\mathrm{G}(\mathrm{V}=0, \mathrm{~F})$ function of $\mathrm{Cu}$ nanowires.

electronic distributions are carried out by the Mulliken charge analysis. $^{15}$

Fig. 1 shows two representative structures of $\mathrm{Cu}$ NWs with nonhelical and helical structures. The structures are referred to as 6-1a and 6-1b where 6 show the atomic number of one atomic layer in the surface cell and 1 represents that in the core. ${ }^{16}$ The lattice parameter $\mathrm{C}$ denotes the length $(L)$ of $\mathrm{Cu}$ NWs, and $L(V=0, F)$ functions of 6-1a and 6-1b are shown in Fig. 2, where $F$ denotes the stress field strength in nN. It is apparent that $L$ of $6-1 \mathrm{a}$ and $6-1 \mathrm{~b}$ decreases sharply when $F$ increases from 0 to $5 \mathrm{nN}$ and keeps almost constant when $F>5 \mathrm{nN}$. This is because, when $F>5 \mathrm{nN}$, the distances between certain atoms are close to the critical value $(0.23 \mathrm{~nm})$ and repulsive forces dominates the lattice distance. Due to it, there is not enough space for the movement of atoms. According to the inset in Fig. 2, $G(V=0, F)$ of both 6-1a and 6-1b is enhanced as $F$ increases from 0 to 5 $\mathrm{nN}$ but decrease as $F$ is increased further. The trend for $G(V=0, F)$ for both structures are almost the same, which agrees with the trend for $L(V=0, F)$. It is because that the atom accumulation becomes stronger when the distance among some atoms decreases. But when $F>5 \mathrm{nN}$, the stability of structure of $\mathrm{Cu}$ nanowire is broken and positions of atoms are out of order, so $G(V=0, F)$ goes down to zero while $L(V=0, F)$ keeps constant.

According to the Landauer formula, the number of bands crossing Fermi level $E_{\mathrm{f}}$ attributes to the number of conductional channels or the magnitude of $G$. The calculated $G(V=0, F=0)$ and $G(V=1, F=0)$ values of 6-1a and 6-1b with all conductional channels are shown in Figs. 3(a) and 3 (b) and Table I. $G(V=0, F=0)=4$ agrees with other results. ${ }^{13}$ As $V$ further increases, $G(V, F=0)$ of $6-1 \mathrm{a}$ decreases and $G(V=1, F=0) / G(V=0, F=0)=0.5$, but $G(V, F=0)$ of $6-1 \mathrm{~b}$ increases and $G(V=1) / G(V=0)=1.5$. $G(V, F=0)$ functions of the both structures indeed have opposite change tendencies on $L(V, F=0)$.

For 6-1a, $L(V, F=0)$ reaches the maximum at $V=0.5$ where the atomic structure varies and distance between layers $(D)$ in the middle of 6-1a increases. The increase of $D_{4-6}$ decreases the electron jump between the two layers due to the ballistic transport nature and thus decreases $G$. When $V=1, D_{4-6}$ is about 1.5 times of that at $V=0$ as shown in Fig. 1 and Table I. According to the above elucidation,

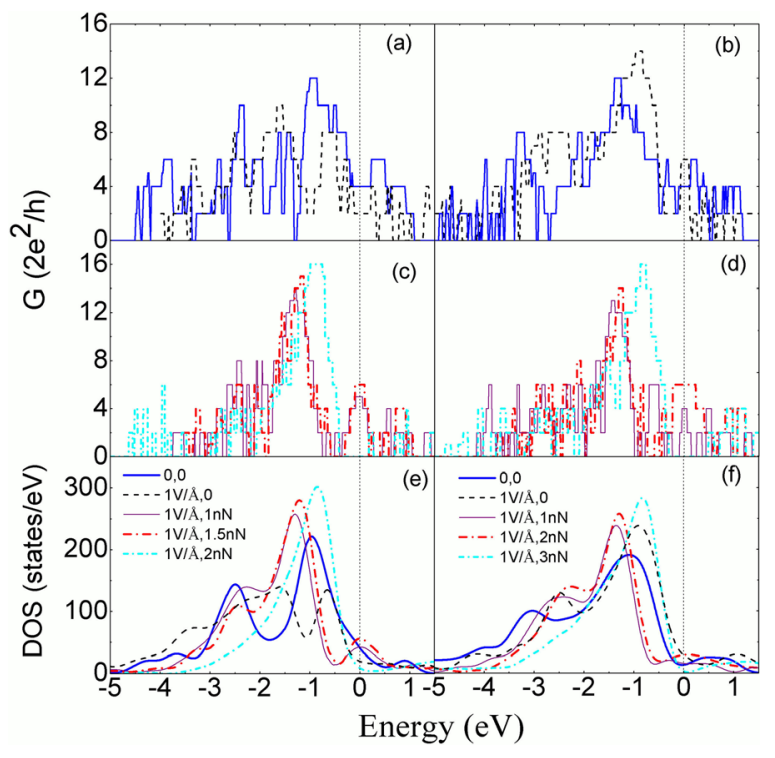

FIG. 3. (Color online) $G(V, \mathrm{~F})$, DOS of Cu NWs with 6-1a of (a), (e), (g), (i), and (k) and with 6-1b of (b), (f), (h), (j), and (1) in Figure 1. $E_{\mathrm{f}}=0$ (vertical dotted line) is taken.

$L(V=0, F)$ achieves constant when $F>5 \mathrm{nN}$. However, when $V=1$, the critical force for structural collapses are reduced to $2 \mathrm{nN}$, which means the role of $V$ for $6-1$ a is similar to a strong $F$. Meanwhile, when $V=1$ and $F=1.5 \mathrm{nN}$, $G$ increases obviously and $G(V=1, F=1.5 \mathrm{nN}) / \mathrm{G}(V=1$, $F=0)=3$. When $V=1$ and $F<2 \mathrm{nN}, G$ increases as $F$ increase. However, when $V=1$ and $F=2 \mathrm{nN}, G$ decreases sharply because the structure collapses.

Similar to 6-1a, when $F=0$ and $V \geq 0.5, L(V)$ functions of 6-1b drop monotonously, but $D(V)$ of the middle layers increase a little. When $F=0, V=1$, where the atoms in the odd layers go to the interstice of the next layer and the structure tends to continuously shrink. Similar to 6-1a, atoms also accumulate on both tips of NWs, while the atomic number in the middle part of NWs decreases. When $V$ is equal to $1 \mathrm{~V} / \AA$ and $F \neq 0$, atoms no longer accumulate on both tips of NWs. When $F=1 \mathrm{nN}$ and $V=1 \mathrm{~V} / \AA$, the atoms in the odd layers no longer go to the interstice of the next layer. Moreover, $G$ is equal to 4 and smaller than the value at $F=0$ and $V=1 \mathrm{~V} /$ $\AA$. This is due to the fact that the structure does not shrink continuously, but trends to the stable status. It looks like the structure of 6-1b without any external fields, which can be understood as some type of counteraction. When $F=2 \mathrm{nN}$ and $V=1 \mathrm{~V} / \AA$, $G$ increases from 4 to 6 and the structure is comparatively stable than the structure at $F=0$ and $V=1 \mathrm{~V} /$ $\AA$. However, when $F=3 \mathrm{nN}$ and $V=1 \mathrm{~V} / \AA$, $G$ decreases to 0 because the structure collapses. The critical pressure of collapse of $6-1 \mathrm{~b}$ is higher than $F=2 \mathrm{nN}$ for $6-1 \mathrm{~b}$. $6-1 \mathrm{~b}$ could stand more $F$ than 6 -1a and would collapse under higher force intensity when $V=1 \mathrm{~V} / \AA$.

TABLE I. $G(V)$ function of $\mathrm{Cu}$ nanowires obtained from DFT calculations. $G$ in $G_{0} . V$ in $\mathrm{VA}^{-1} . F$ in nN.

\begin{tabular}{lcccccc}
\hline \hline & & & & & & \\
& $F=0$, & $V=1$, & $V=1$, & $V=1$, & $V=1$, & $V=1$, \\
& $F=0$ & $F=1$ & $F=1.5$ & $F=2$ & $F=3$ \\
\hline$G(6-1 \mathrm{a})$ & 4 & 2 & 4 & 6 & 0 & 0 \\
$G(6-1 \mathrm{~b})$ & 4 & 6 & 4 & 5 & 6 & 0 \\
\hline \hline
\end{tabular}



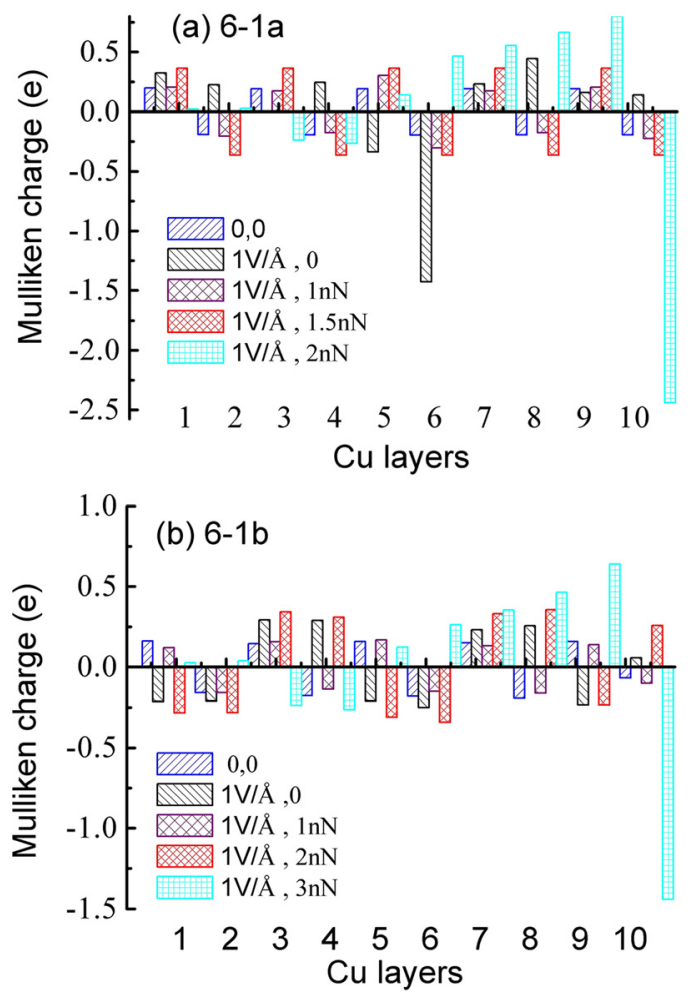

FIG. 4. (Color online) The Mulliken charge population of 6-1a (a) and 6-1b (b).

DOS of the two structures obtained DFT calculations are presented in Figs. 3(e) and 3(f). When $F=0$, for both of 6-1a and b-1b, the largest peak obviously shifts right as $V$ increases, which implies the energy increases of both structures. However, when $F>0$, the trend of $G$ is totally different. The largest peak obviously shifts left when $F$ is applied, which implies that the energy decreases of both structures and the structure becomes more stable. When $V$ keeps constant, the largest peak obviously shifts left as $F$ increases. While $F=3 \mathrm{nN}$, the magnitude of DOS at $E_{\mathrm{f}}$ decreases to 0.5 times of that under $F=0$ for $6-1 \mathrm{a}$ and the value is 0.75 times for $6-1 \mathrm{~b}$ as the structure collapses. In the same way, the above results for DOS ratios at $E_{\mathrm{f}}$ confirm the calculated results from Landauer formula shown in Figs. 3(c) and 3(d), where the corresponding $G$ ratios are 0.5 and 1.5. Meanwhile, whether the external electric field and stress field are applied or not, $G$ are always mainly dominated by the charge distribution in the inner atomic layer which should be attributed to the reason that the electron scattering may hold back the electron transport at the surface layers induced by the lattice contraction there. ${ }^{3,16}$

$e(V)$ functions of 6-1a and 6-1b are shown in Figs. 4(a) and 4(b). The layer is indexed in Fig. 1. When $F=0$, under $V=0, e(V)$ functions of the both structures are similar and homogeneous along the axis of NWs. When $V=1, e$ reaches -1.4 . Now the electron accumulation occurs only in the 5 th and 6th layers while charges in all other layers are positive. This accumulation is unfavorable for the electronic transport. However, When $F=1.5 \mathrm{nN}$ and $V=1$, the electron accumulation totally disappears, the value of $e(1.5 \mathrm{nN}, 1)$ (including positive and negative charges) in each layers are richer than $e(0,0), e(0,1)$, and $e(1 \mathrm{nN}, 1)$. However, when $F=2 \mathrm{nN}$ and $V=1$, the symmetry distribution of charge is broken, the charge is out-of-order. The electron transport is broken off, and the electron accumulation is thus observed in the 10th layers.

In the case of 6-1b, electrons are equably distributed in all layers under $V$. The positive and negative charges are alternately distributed; however, it is not from one, but two layers. This is directly related to the fact that the odd and even layers combine and form a new layer consisting of seven $\mathrm{Cu}$ atoms, which increases the channel numbers of ballistic transport. However, when $F=1 \mathrm{nN}$ and $V=1$, the new layer shown above disappears, even it does not benefit electronic transport, but the total energy decreases and the structure trends to be more stable. When $F=2 \mathrm{nN}$ and $V=1$, the above so-called new layer appears again; the value of $e(2$ $\mathrm{nN}, 1)$ (including positive and negative charges) in each layers are richer than $e(0,0), e(0,1)$, and $e(1.0 \mathrm{nN}, 1)$. However, when $F=3 \mathrm{nN}$ and $V=1$, the distribution of charge is out-of-order. The electrons transport is broken off and the electron also accumulates in the 10th layers, just like the results of 6-1a under $F=2 \mathrm{nN}$.

In summary, we studied the effect of electric and stress field on atomic and electronic structures and transport properties of $\mathrm{Cu}$ NWs with nonhelical and helical structures. When the stress field is considered, both structures are more stable than those only under electric field. $G(V, F=0)$ function of 6$1 \mathrm{~b}$ increases as $V$ increases, but $G(V, F=0)$ of 6-1a has a reverse direction. $G(V=1, F)$ function of 6-1a increases as $F$ increases when $0<F<1.5 \mathrm{nN}$, while $G(V=1, F)=0$ when $F>1.5 \mathrm{nN}$, and $6-1 \mathrm{~b}$ has a similar trend. The helical atomic strands are more stable than the nonhelical ones since the former owns higher collapse-resistant $F$.

We acknowledge the supports by National Key Basic Research and Development Program (Grant No. 2010CB631001) and the Fundamental Research Funds for the Central Universities.

\footnotetext{
${ }^{1}$ A. R. Rathmell, S. M. Bergin, Y. L. Hua, Z. Y. Li, and B. J. Wiley, Adv. Mater. 22, 3558 (2010).

${ }^{2}$ S. Y. Chang and D. S. Chen, Appl. Phys. Lett. 94, 231909 (2009).

${ }^{3}$ Y. F. Zhu, X. Y. Lang, W. T. Zheng, and Q. Jiang, ACS Nano 4, 3781 (2010).

${ }^{4}$ L. Olesen, E. Laegsgaard, I. Stensgaard, F. Besenbacher, J. Schiotz, P. Stoltze, K. W. Jacobsen, and J. K. Norskov, Phys. Rev. Lett. 72, 2251 (1994).

${ }^{5}$ B. J. Van Wees, H. Van Houten, C. W. J. Beenakker, J. G. Williamson, L. P. Kouwenhoven, D. Van der Marel, and C. T. Foxon, Phys. Rev. Lett. 60, 848 (1998).

${ }^{6}$ G. E. Tommei, F. Baletto, R. Ferrando, R. Spadacini, and A. Danani, Phys. Rev. B 69, 115426 (2004).

${ }^{7}$ J. J. Zhao, C. Buia, J. Han, and J. P. Lu, Nanotechnology 14, 501 (2003).

${ }^{8}$ C. Q. Sun, H. L. Bai, S. Li, B. K. Tay, C. Li, T. P. Chen, and E. Y. Jiang, J. Phys. Chem. B 108, 2162 (2004).

${ }^{9}$ E. Tosatti, S. Prestipino, S. Kostlmeier, A. Dal Corso, and F. D. Di Tolla, Science 291, 288 (2001).

${ }^{10}$ O. Gulseren, F. Ercolessi, and E. Tosatti, Phys. Rev. Lett. 80, 3775 (1998).

${ }^{11}$ J. P. Perdew, K. Burke, and M. Ernzerhof, Phys. Rev. Lett. 77, 3865 (1996).

12Z. M. Ao and F. M. Peeters, Appl. Phys. Lett. 96, 253106 (2010).

${ }^{13}$ B. L. Wang, J. J. Zhao, X. S. Chen, D. Shi, and G. H. Wang, Nanotechnology 17, 3178 (2006).

${ }^{14}$ D. Sanchez-Portal, E. Atacho, and J. M. Soler, Solid State Commun. 95, 685 (1995).

${ }^{15}$ W. Liu, Y. H. Zhao, J. Nguyen, Y. Li, Q. Jiang, and E. J. Lavernia, Carbon 47, 3452 (2009).

${ }^{16}$ Y. F. Zhu, W. T. Zheng, and Q. Jiang, Appl. Phys. Lett. 95, 083110 (2010).
} 